\title{
La forestación de tierras agrícolas: balance de un instrumento de política forestal para el cambio del uso de la tierra
}

\author{
Afforestation of agricultural lands: \\ balance of a forest policy instrument for land use change
}

Vadell, E. '; de-Miguel, S. ${ }^{2}$; Fernández Centeno, G. ; Robla, E.;

Lerner, M. ${ }^{4}$ Pemán, J. ${ }^{*}$

${ }^{1}$ Generalitat de Catalunya, Departament d'Agricultura, Ramaderia, Pesca i Alimentació,

Merce Rodoreda 16, La Seu d'Urgell, 25700, Lleida.

${ }^{2}$ Departamento de Producción Vegetal y Ciencia Forestal, Universidad de Lleida-Agrotecnio Center, Rovira Roure 191, 25198, Lleida.

${ }^{3}$ Subdirección General de Política Forestal, Dirección General de Desarrollo Rural, Innovación y Politica Forestal, Ministerio de Agricultura, Pesca y Alimentación, Gran Vía de San Francisco 4 y 6, 28005, Madrid

${ }^{4}$ TRAGSATEC, Julián Camarillo 6, 28037, Madrid

Autor para correspondencia: peman@pvcf.udl.cat 


\title{
Resumen
}

Se han cumplido 25 años del inicio del programa de forestación de tierras agrarias con motivo de la reforma de la Política Agrícola Comunitaria (PAC) en 1992. El objetivo de este estudio es realizar un análisis global, a nivel de Comunidad Autónoma, que permita identificar los elementos más característicos del programa, como paso previo a futuros análisis más detallados. A partir del conocimiento del marco normativo se analiza la superficie forestada, las especies utilizadas, el tipo de tierras forestadas y su superficie media. La superficie total forestada ha superado las 730 mil hectáreas. Castilla y León ha forestado el 35\% de la superficie total . El 65\% de esta superficie se forestó en el periodo 1993-99 y solo un 8\% a partir de 2007. El comportamiento de la superficie forestada por cada Comunidad Autónoma, en el periodo 1993-99, ha mostrado una relación con la superficie total de cultivos existentes en cada comunidad al inicio del programa. El 15\% de la superficie forestada fue con especies de crecimiento rápido, el 50\% de masas monoespecíficas de especies de crecimiento lento, con un equilibro entre el uso de coníferas y frondosas y el resto correspondió a masas mixtas con un predominio de frondosas. Las tierras forestadas correspondieron, mayoritariamente, a cultivos herbáceos de secano o a eriales a pastos. La superficie media fue de 12 hectáreas, con una gran variabilidad entre Comunidades Autónomas. La forestación de tierras agrarias ha sido el programa de mayor impacto en España de ayudas a la repoblación a los propietarios particulares. Sus resultados han sido muy heterogéneos en las diferentes Comunidades Autónomas y, hoy por hoy, insuficientemente documentados y estudiados, por lo que se precisa que se intensifiquen los estudios sobre la misma.

Palabras clave: ayudas forestales europeas, plantaciones de frondosas y coníferas, plantaciones mixtas, plantaciones especies crecimiento rápido, eriales a pastos.

\begin{abstract}
Twenty-five years have passed since the beginning of the afforestation of agricultural lands program, in 1992, resulting from the reform of the Common Agricultural Policy (CAP). The objective of this study is to carry out a thorough analysis by Spanish Autonomous Communities, allowing for identifying the key features of that program, as a preliminary step to further in-depth analysis and research. Based on the knowledge of the regulatory framework, we analyze the forested area, the species used, the type of forested land and the average forested area. The total forested area has exceeded 730 thousand hectares. Sixty five percent of this area was afforested during the period 1993-99, and only 8\% from 2007 onwards. The trend of the afforested area by Autonomous Community during the period 1993-99 was related to the total area of existing crops in each Autonomous Community at the beginning of the program. Thus, Castilla y León region has afforested 35\% of the total afforested area. Fast-growing species represented 15\% of the total afforested land in Spain, while monospecific stands of slow-growing species represented $50 \%$ of the total afforested area, with a balanced use of conifers and hardwoods, and the rest corresponded to mixed stands with hardwoods as predominant species. The afforested lands were mostly areas previously devoted to arable crops or uncultivated lands for pastures. The average afforested area was 12 hectares, with great variability between Autonomous Communities. The afforestation of agricultural lands has been the program with the greatest impact in Spain in terms of support to the afforestation of private ownerships. The outcomes of this program have been very heterogeneous among different Spanish regions and, so far, they have been poorly documented and studied. Further research is therefore needed.
\end{abstract}

Keywords: European forestry aids, plantations of broadleaves and conifers, mixed plantations, plantation of fastgrowing species, marginal grazing land. 


\section{Introducción}

El Programa de Forestación de Tierras Agrarias (FTA) surgió como consecuencia de la reforma realizada por la Comunidad Económica Europea en 1992 de la Política Agrícola Común. La FTA fue una medida forestal que se insertaba dentro de unas medidas de acompañamiento y que iba dirigida principalmente a los titulares de explotaciones agrícolas. Existen diferentes estudios, en cuanto a su ámbito temporal y territorial, sobre la evolución de la FTA a lo largo de los 25 años que lleva en vigor. A nivel estatal, destacan los trabajos de Gómez-Jover y Jiménez Peris (1997), que analizaron el periodo 1993-95 o el del MAPA (2006) que analizó el periodo 1994-2006. A nivel de Comunidad Autónoma, Rodríguez-Martínez (1993) analizó el periodo 1994-2008 en Castilla-La Mancha, Álvarez (2004) estudió el periodo 1996-2002 en Galicia, González-Botía (2017) analizó las forestaciones realizadas entre 1993-99 en Murcia y Sequeda (2017) analizó el periodo 1993-2016 en Extremadura. A día de hoy, no se dispone, por tanto, de un estudio con un ámbito estatal que analice la FTA a lo largo de todo el periodo que lleva vigente.

Los objetivos de este trabajo, por tanto, son los siguientes:

- Analizar el marco legal que ha desarrollado la FTA a nivel de la Unión Europea (UE), del Estado y de las CC.AA.

- Analizar el modelo de subvención y las superficies objeto de forestación.

- Analizar, a nivel estatal, la superficie forestada, las especies utilizadas, el uso previo de los terrenos forestados y la superficie media forestada por cada beneficiario.

- Analizar que variables pueden explicar la superficie forestada en cada Comunidad Autónoma.

\section{Material y métodos}

Se analizó la legislación dictada por los organismos europeos y los desarrollos que han tenido a nivel estatal y autonómico. Para recabar el resto de la información se consultaron los siguientes documentos

- Anuarios de estadística forestal (MAPA, 2016).

- Evaluation for the Community aid scheme for forestry measures in agriculture Regulation N 2080/92 (1993-1999) (Picard, 2001).

- Informe final del Estado del Programa de Desarrollo Rural del periodo 20002006 (TRAGSATEC, 2008).

- Informe final de los Programas de Desarrollo Rural de las Comunidades Autónomas para el periodo 2007-2013 (MAPA, 2013).

- Estadística de la FTA de la Junta de Castilla-La Mancha (JCLM, 2012).

— Estadística de la FTA de la Junta de Castilla y León (JCyL, 2018).

- Estadística de la FTA del Gobierno de Canarias (CACAN, 2019).

El análisis realizado se estructura en las siguientes etapas: 
1) Marco legal y financiero. Se analizó su variación a lo largo de los diferentes periodos de programación, describiéndose, en particular, el sistema de subvención y las superficies objeto de forestación.

2) La superficie forestada.

3) Las especies utilizadas en la forestación.

4) El tipo de tierras agrarias forestadas.

5) La superficie media forestada por beneficiario.

\section{Resultados y discusión}

\subsection{Marco legal y financiero}

La FTA ha tenido un marco legal que ha evolucionado durante los 25 años que lleva vigente, adaptándose a los objetivos perseguidos en los diferentes periodos de programación:

- Período 1993-99. Se corresponde con la Reforma de la Política Agraria Común (PAC), de mayo de 1992, que incluía unas medidas de acompañamiento que hacían referencia a tres aspectos:

- Métodos de producción agraria compatibles con las exigencias del medio ambiente.

- Medidas forestales.

- Jubilación anticipada de los agricultores.

Las medidas forestales se concretaron en dos reglamentos europeos:

- El Reglamento CEE 2080/92 sobre un régimen comunitario de ayudas a las medidas forestales en la agricultura.

- El Reglamento CEE 1610/89 por el que se establecen las normas para la acción de desarrollo y aprovechamiento de los bosques en las zonas rurales de la Comunidad. En España se aplicaron mediante el RD. 378/93 régimen de ayudas para fomentar las inversiones forestales en explotaciones agrarias y acciones de desarrollo y aprovechamiento de los bosques en zonas rurales y, posteriormente, mediante el RD 152/96 régimen de ayudas para fomentar las inversiones forestales en explotaciones agrarias y acciones de desarrollo y aprovechamiento de los bosques en zonas rurales. Los objetivos perseguidos con estas ayudas eran que las nuevas forestaciones fueran un complemento de renta para los agricultores, además de contribuir a la creación de masas forestales con una superficie mínima que permita su gestión racional.

- Período 2000-06. La Conferencia de Cork sobre desarrollo rural, en 1996, planteó un sector agrícola europeo de carácter multifuncional y en un contexto social y territorial más amplio. El marco normativo lo desarrolló el Reglamento 1257/1999, sobre ayudas al desarrollo rural a cargo del Fondo 
Europeo de Orientación y Garantía Agrarias (FEOGA-Garantía), que añadió a las tres medidas de acompañamiento del anterior reglamento dos más: el de las zonas desfavorecidas y el de zonas con restricciones ambientales. Su aplicación en España se realizó mediante 20 programas, 17 de carácter regional y 3 de carácter pluriregional. Entre estos últimos estaba el Programa Horizontal de Desarrollo Rural para las Medidas de Acompañamiento. Dentro de este programa no participaron ni País Vasco ni Navarra, debido a sus diferentes regímenes fiscales. La FTA se constituyó, en este nuevo marco, en un instrumento cuyo objetivo principal era el de aumentar la superficie forestal. Las CC.AA. de Islas Baleares, Comunidad Valenciana, Canarias, Cataluña y Región de Murcia no activaron la FTA. En España se reguló este instrumento mediante el RD 6/2001, de 12 de enero, sobre el fomento de la forestación en tierras agrícolas. Los objetivos que se pretendían con estas ayudas, como así recogía el RD 6/2001, eran: diversificar la actividad agraria, generando fuentes alternativas de renta y de empleo, contribuir a la corrección de los problemas de erosión, desertización y a la conservación y mejora de los suelos.

- Periodo 2007-13. El Reglamento (CE) 1698/2005, del Consejo, relativo a la ayuda al desarrollo rural a través del Fondo Europeo Agrícola de Desarrollo Rural (FEADER), estableció una programación basada en un Plan Estratégico Nacional (PEN), que recogiera las prioridades de esta política de cada Estado miembro. España, debido a su descentralización administrativa, decidió crear un marco planificador en el que se integraran los programas de desarrollo rural de las CC.AA. Siguiendo estos criterios, se elaboró el Programa Nacional de Desarrollo Rural (2007-2013) que contempló unas medidas horizontales derivadas de un Marco Nacional de Desarrollo Rural y otras medidas más específicas para cada comunidad autónoma Las CC.AA. fueron libres de elegir las medidas para el cumplimiento de sus objetivos. La FTA quedó englobada en el eje relativo a la Mejora del medio ambiente y del entorno rural y, dentro de él, en la medida relativa a la utilización sostenible de las tierras forestales a través de ayudas a la primera forestación de tierras agrícolas. También contemplaron ayudas a la primera forestación de tierras no agrícolas. En el año 2009, como consecuencia de la aprobación del Reglamento (CE) 74/2009, que modificaba el Reglamento (CE) 1698/2005, la FTA apareció, también, como instrumento para la adaptación al cambio climático y mitigación del cambio climático y a la protección y mejora de la calidad del agua. La FTA, por tanto, se convirtió en una medida elegible para el cumplimiento de diferentes objetivos.

- Periodo 2014-20. El marco legal es el Reglamento UE 1305/2013, de 17 de diciembre de 2013, relativo a la ayuda al desarrollo rural a través del Fondo Europeo Agrícola de Desarrollo Rural (FEADER). La FTA y la reforestación se contemplan como unas medidas de especial relevancia para restaurar, conser- 
var y mejorar los ecosistemas dependientes de la agricultura y la silvicultura y para mejorar la eficiencia de los recursos y apoyar el paso a una economía baja en emisiones de carbono y adaptable al cambio climático en los sectores agrario, alimentario y forestal. En España se presentó el Programa Nacional de Desarrollo Rural, a nivel estatal, y 17 PDR autonómicos. Paralelamente a este reglamento, la Comisión aprobó en 2014, el Reglamento UE 702/2014, de 25 de junio, por el que se declaran determinadas categorías de ayuda en los sectores agrícola y forestal y en zonas rurales compatibles con el mercado interior en aplicación de los artículos 107 y 108 del Tratado de Funcionamiento de la Unión Europea. Dentro de estas ayudas se contempla la reforestación y la creación de superficies forestales en terrenos agrícolas y no agrícolas.

Hay que tener presente, al hablar del marco legal de este programa, que las CC.AA. han desarrollado particularmente cada una de estas normativas, por lo que puede haber ciertas diferencias en su aplicación territorial. Atendiendo a las nomas actualmente en vigor, y que se corresponden con este periodo de programación, se puede observar que la FTA continúa siendo una ayuda elegible en el Principado de Asturias, Castilla-La Mancha, Castilla y León, Diputación Foral de Álava, Región de Murcia y La Rioja.

\subsubsection{El sistema de subvención}

Una de las innovaciones que supuso la forestación de tierras agrarias fue su modelo de subvención, compuesto de tres elementos:

- Coste de implantación o establecimiento.

- Prima de mantenimiento durante los primeros cinco años.

- Prima compensatoria de renta. Esta prima se estableció inicialmente durante 20 años, para irse reduciendo a 15 y finalmente a 12 años. El Reglamento 1305 / 2013 integró la prima de mantenimiento y la compensatoria en una única prima para un periodo máximo de doce años. En el caso de que los beneficiarios fueran entidades públicas, la subvención solo cubría los gastos de establecimiento.

La prima compensatoria fue el elemento más atractivo del sistema, por su carácter de ingreso garantizado durante 20 años. Esta prima, sin duda, atrajo a numerosos agricultores, aunque luego ha sido el elemento que ha condicionado a este programa, por el coste tan elevado que ha supuesto. Su coste ha superado, en todos los proyectos financiados, el 50\% del coste total del mismo, llegando, en algunos casos, a superar el 70\% (UE, 2005).

El coste de estas primas dependía de las especies que se utilizaban. Así, entre 1993 y 2001 las especies se clasificaron en tres grupos:

- Anexo I: Especies arbóreas cuya plantación tenga como fin principal la producción de madera a un plazo mayor de quince años. Agrupa, entre otras, a 
las siguientes especies: Pinus sylvestris, P.uncinata, P.nigra, P.pinaster, P.pinea, P.halepensis, P.canariensis, P.radiata, Cupresus spp., Cedrus spp., Larix spp., Quercus rubra, Platanus spp., etc. Sorprende, sobremanera, el objetivo asignado a especies como P.uncinata, P.pinea, P.halepensis y P.canariensis.

- Anexo II: Especies arbóreas y arbustivas cuya plantación tenga como fin principal la restauración o la creación de masas forestales permanentes. Agrupa, entre otras, a las siguientes especies: Abies alba, Populus alba, P.nigra, P.tremula, Salix alba, S.fragilis, Betula pendula, Alnus glutinosa, Ulmus minor, U.pumila, U.glabra, Acer spp., Tilia spp., Olea europea, Quercus robur, Q.petraea, Q.pyrenaica, Q.faginea, Q.lusitanica, Q.suber, Q.ilex, Tamarix spp., Buxus sempervirens, etc.

- Anexo III: Especies arbóreas y arbustivas autóctonas de interés particular en ciertas zonas por motivos de producción de maderas valiosas, endemismos, peligro de extinción, etc. Agrupa a las siguientes especies: Juniperus phoenicea, J.thurifera, Taxus baccata, Juglans regia, Arbutus unedo, Tetraclinis articulata, Prunus spinosa, P.insititia e Ilex aquifolium.

E1 RD 6/2001 clasificó las especies en 5 grupos:

- Frondosas.

- Frondosas de crecimiento lento masa pura.

- Frondosas de crecimiento lento masa mezclada.

- Frondosas de crecimiento rápido raíz profunda.

- Frondosas de crecimiento rápido raíz superficial.

- Resinosas.

- Resinosas de crecimiento lento masa pura.

- Resinosas de crecimiento lento masa mezclada.

- Resinosas de crecimiento rápido. Por resinosas se entiende a las gimnospermas, que abarcan las coníferas y las taxáceas.

- Mezclas de frondosas y resinosas. Se distinguen:

- Crecimiento lento.

- Crecimiento rápido.

- Otras arbóreas de crecimiento lento (laurisilva, etc.).

- Arbustivas

Las diferencias de primas según la especie fue muy relevante (tabla 1). En el periodo 1993-99 la prima de establecimiento para las especies del Anexo II y III fue- 
ron un 71 y $86 \%$ superiores, respectivamente, a las especies del Anexo I. Las primas de mantenimiento lo fueron en un 33 y $100 \%$ y la prima compensatoria en 40 y $75 \%$. Esta diferencia, sin duda, influyó en las especies elegidas para la forestación, aunque hay que tener en cuenta que la elección de la especie, como otras decisiones de carácter técnico, debía contar con la aprobación de la administración.

Tabla 1. Primas establecidas por especie.

\begin{tabular}{|c|c|c|c|c|}
\hline \multirow[t]{2}{*}{ Norma } & \multirow[t]{2}{*}{ Especies } & \multicolumn{3}{|c|}{ Importe máximo $\left(€\right.$ ha'-1 $^{-1}$} \\
\hline & & Establecimiento & Mantenimiento & Compensación \\
\hline \multirow[t]{4}{*}{ RD. 378/931 } & Anexo I & $1052^{2}$ & 90 & $120^{6}$ \\
\hline & Anexo II & 1803 & $120^{4}$ & $168^{6}$ \\
\hline & Anexo III & $1953^{3}$ & $180^{5}$ & $210^{6}$ \\
\hline & $\begin{array}{l}\text { Crecimiento rápido } \\
\text { en turno corto }(<15 \text { años })\end{array}$ & 721 & & \\
\hline \multirow[t]{4}{*}{ RD 152/96 } & Anexo I & $1097^{2}$ & 150 & $138^{6}$ \\
\hline & Anexo II & 1881 & $180^{4}$ & $192^{6}$ \\
\hline & Anexo III & $2037^{7}$ & $240^{5}$ & $240^{6}$ \\
\hline & $\begin{array}{l}\text { Crecimiento rápido } \\
\text { en turno corto }(<15 \text { años })\end{array}$ & 751 & & \\
\hline \multirow[t]{11}{*}{ RD 6/2001 } & $\begin{array}{l}\text { Frondosas de crecimiento } \\
\text { lento masa pura }\end{array}$ & 2314 & 288 & 325 a $60^{8}$ \\
\hline & $\begin{array}{l}\text { Frondosas de crecimiento } \\
\text { lento masa mezclada }\end{array}$ & 2560 & 288 & \\
\hline & $\begin{array}{l}\text { Frondosas de crecimiento } \\
\text { rápido raíz profunda }\end{array}$ & 1839 & & \\
\hline & $\begin{array}{l}\text { Frondosas de crecimiento } \\
\text { rápido raíz superficial. }\end{array}$ & 1352 & & \\
\hline & $\begin{array}{l}\text { Resinosas de crecimiento } \\
\text { lento masa pura }\end{array}$ & 1262 & 180 & 313 a $54^{8}$ \\
\hline & $\begin{array}{l}\text { Resinosas de crecimiento } \\
\text { lento masa mezclada }\end{array}$ & 1382 & 180 & \\
\hline & Resinosas de crecimiento rápido & 1226 & & \\
\hline & $\begin{array}{l}\text { Mezclas de frondosas } \\
\text { y resinosas de crecimiento lento }\end{array}$ & 1779 & 210 & 337 a $60^{8}$ \\
\hline & $\begin{array}{l}\text { Mezclas de frondosas } \\
\text { y resinosas de crecimiento rápido }\end{array}$ & 1424 & & \\
\hline & Otras arbóreas crecimiento lento & 2855 & 288 & 355 a $186^{9}$ \\
\hline & Arbustivas & 1557 & 90 & 108 a $54^{10}$ \\
\hline $\begin{array}{l}\text { Reglamento } \\
\text { CE } 1698 / 2005\end{array}$ & & $\begin{array}{c}70 \% \text { costes } \\
\text { subvencionables }\end{array}$ & & $\begin{array}{l}700^{11} \\
150^{12}\end{array}$ \\
\hline
\end{tabular}

${ }^{1}$ Para cada titular individual, variando para titulares agrupados. ${ }^{2}$ Se admiten mezclas con las especies del resto de los anejos incrementándose la prima en $150 €$ por cada $25 \%$ de presencia de estas especies. ${ }^{3} \mathrm{Si}$ se foresta con especies de los anejos II y III en un espacio natural protegido la prima asciende a $2404 € €^{4}$ Especies anexos II y III con un máximo del $25 \%$ del anexo I. ${ }^{5}$ Especies anexos II y III. ${ }^{6}$ Para las primeras 25 ha ${ }^{7}$ Si se foresta con especies de los anejos II y III en un espacio natural protegido la prima asciende a $2506 €^{8}$ Varía según el tipo de superficie sean desde tierras de cultivos a erial a pastos. ${ }^{9}$ Varía según el tipo de superficie sean desde tierras de cultivos a pastizales. ${ }^{10}$ Varía según el tipo de superficie sea barbecho o erial a pastos. ${ }^{11}$ Agricultores o asociaciones agricultores. ${ }^{12}$ Cualquier otra persona física o jurídica de derecho privado. 
En el segundo periodo de programación estas diferencias continuaron en las dos primeras primas y se redujo muy significativamente en la prima compensatoria. Así, la prima de establecimiento fue un $83 \%$ superior para la creación de masas puras de frondosas que para la creación de masas puras de resinosas y la prima de mantenimiento lo fue en un $60 \%$. Sin embargo, la prima compensatoria tan solo fue un $4 \%$ superior.

En la actualidad, las primas que se establecen al amparo de la legislación de las CC.AA. presentan variaciones menos relevantes según la especie elegida (tabla 2).

Tabla 2. Primas establecidas por especie en la actualidad.

\begin{tabular}{|c|c|c|c|c|}
\hline Norma & Especies & \multicolumn{3}{|c|}{ Importe máximo $\left(€\right.$ ha $\left.^{-1}\right)$} \\
\hline \multirow{4}{*}{$\begin{array}{c}\text { ORDEN } \\
\text { FYM/399/2015, } \\
\text { de la Comunidad } \\
\text { de Castilla y León }\end{array}$} & & Establecimiento & Mantenimiento & Compensación \\
\hline & Coníferas $(<25 \%$ frondosas $)$ & 2300 & \multirow{3}{*}{130 y $305^{1}$} & \multirow{3}{*}{80 y $400^{2}$} \\
\hline & $\begin{array}{c}\text { Mezcladas }(26 \%<\text { coníferas } \\
\text { y frondosas }<74 \%)\end{array}$ & 2400 & & \\
\hline & Frondosas $(<25 \%$ coníferas $)$ & 2500 & & \\
\hline \multirow{7}{*}{$\begin{array}{c}\text { Orden } 28 / 2017 \text {, } \\
\text { de } 16 \text { de febrero y } \\
\text { Orden de } 15-05- \\
2008 \text { de Castilla-La } \\
\text { Mancha }\end{array}$} & $\begin{array}{l}\text { Frondosas crecimiento lento } \\
\text { densidad } 600 \text { pies }^{-1} \text { ha }^{-1}\end{array}$ & & \multirow{6}{*}{230} & \multirow{7}{*}{$150^{3}$ ó $330^{4}$} \\
\hline & $\begin{array}{l}\text { Frondosas de crecimiento } \\
\text { lento micorrizadas } \\
\text { con Tuber melanosporum, } \\
\text { densidad } 278 \text { pies ha }^{-1}\end{array}$ & 1250 & & \\
\hline & $\begin{array}{l}\text { Juglans regia y Prunus } \\
\text { avium, densidad } 278 \text { pies } \text { ha }^{-1}\end{array}$ & 1500 & & \\
\hline & $\begin{array}{c}\text { Frondosas de crecimiento } \\
\text { rápido a raíz profunda sin } \\
\text { destoconado, densidad } 278 \\
\text { pies ha-1 }\end{array}$ & 1400 & & \\
\hline & $\begin{array}{l}\text { Coníferas de crecimiento } \\
\text { lento, densidad } 800 \text { pies }^{-1}{ }^{-1}\end{array}$ & 1100 & & \\
\hline & $\begin{array}{l}\text { Masas mixtas frondosas } \\
\text { y coníferas, densidad } \\
700 \text { pies ha } \text { ha }^{-1}\end{array}$ & 1200 & & \\
\hline & Especies arbustivas & 1075 & 145 & \\
\hline \multirow{2}{*}{$\begin{array}{l}\text { ORDEN de } 28 \text { de } \\
\text { diciembre de } 2015 \text {, } \\
\text { Xunta de Galicia }\end{array}$} & Coníferas & 1853 & \multirow{2}{*}{ - } & \multirow{2}{*}{ - } \\
\hline & Frondosas & 2398 & & \\
\hline
\end{tabular}

${ }^{1}$ Según el número de orden. ${ }^{2}$ Según tipo de beneficiario, especie y tierra objeto de forestación. ${ }^{3}$ Resto de beneficiarios. ${ }^{4}$ Agricultor a titulo principal.

\subsubsection{Las superficies objeto de subvención}

Desde el inicio del programa las superficies objeto de esta ayuda han variado (tabla 3). Así, se ha pasado del concepto amplio de tierra agraria, desde 1993 al 
2005, al concepto más estricto de superficie agrícola, desde el año 2005. No obstante, desde ese año, al integrarse esta ayuda en las políticas desarrollo rural se contempla la posibilidad de forestar tierras agrícolas y no agrícolas. Este último término englobó a las tierras abandonadas, los llamados eriales a pastos, que hasta el Reglamento CE 1698/2005 se consideraban tierras agrícolas.

Tabla 3. Concepto de superficie agraria o agrícola cuya forestación estaba subvencionada.

\begin{tabular}{|c|c|}
\hline Norma & Terrenos subvencionables \\
\hline$\frac{\text { RD. } 378 / 93}{\text { RD. } 152 / 96}$ & $\begin{array}{l}\text { Se consideran superficies agrarias, las tierras que hayan sido objeto de una utiliza- } \\
\text { ción agraria en el último decenio y sean susceptibles de forestación. Dichas tierras } \\
\text { serán las comprendidas en alguno de los apartados siguientes: } \\
\text { 1. Tierras ocupadas por cultivos herbáceos (tierras arables). } \\
\text { 2. Barbechos y otras tierras no ocupadas. } \\
\text { 3. Huertos familiares. } \\
\text { 4. Tierras ocupadas por cultivos leñosos (frutales, viñedo, olivar, agrios, etc.). } \\
\text { 5. Prados naturales. } \\
\text { 6. Pastizales. } \\
\text { 7. Los montes de alcornocal. } \\
\text { 8. Monte abierto y dehesas, siempre que las copas del arbolado no cubran más } \\
\text { del } 20 \text { por } 100 \text { de la superficie y se utilice principalmente para pastoreo. } \\
\text { 9. Erial a pastos. }\end{array}$ \\
\hline
\end{tabular}

RD. 6/2001 Tierra agrícola: no estén catastradas como forestales y hayan tenido aprovechamiento agrícola o ganadero de forma regular desde 10 años de la fecha de solicitud.

- Tierras ocupadas por cultivos leñosos.

- Tierras ocupadas por cultivos herbáceos.

- Huertos familiares.

- Prados naturales.

- Pastizales.

- Barbechos.

- Eriales a pastos

\begin{tabular}{ll}
\hline $\begin{array}{c}\text { Reglamento } \\
\text { CE 1698/2005 }\end{array}$ & Tierras agrícolas y no agrícolas \\
\hline Reglamento & $\begin{array}{l}\text { Se contemplan ayudas a la primera forestación de tierras agrícolas y no agrícolas. Tie- } \\
\text { rras agrícolas (cualquier superficie dedicada a tierras de cultivo, pastos permanen- } \\
\text { tes, pastizales permanentes o cultivos permanentes). }\end{array}$
\end{tabular}

La desaparición del objetivo inicial que tuvo la FTA y la libertad otorgada a los Estados miembros en los reglamentos posteriores para determinar las superficies objeto de esta ayuda, ha permitido que las CC.AA. fijen diferentes tipos de superficie (tabla 4).

Como consecuencia de la forestación, las tierras agrarias pasan a estar calificadas a todos los efectos como monte, debiendo reclasificarse en el catastro, a excepción de las forestadas con especies de crecimiento rápido que no precisan de tal reclasificación (RD.6/2001). 
Tabla 4. Superficies objeto de forestación según la normativa de las Comunidades Autónomas .

\begin{tabular}{cc}
\hline Norma & Superficies objetivo de forestación \\
\hline $\begin{array}{c}\text { Orden de 15-05-2008 } \\
\text { de Castilla-La Mancha }\end{array}$ & Cultivos leñosos, huerta, tierra arable \\
\hline $\begin{array}{c}\text { Orden FYM/399/2015, } \\
\text { de la Comunidad de Castilla y León }\end{array}$ & $\begin{array}{l}\text { Pasto con arbolado, pasto arbustivo, pastizal, tierras arables, } \\
\text { huerta, improductivos }\end{array}$ \\
\hline $\begin{array}{c}\text { Orden de 28 de diciembre de 2015 } \\
\text { de la Xunta de Galicia }\end{array}$ & Forestal, Pasto con arbolado, pasto arbustivo \\
\hline $\begin{array}{c}\text { Orden37/2015, de 16 de septiembre } \\
\text { de La Rioja }\end{array}$ & Terrenos rústicos o montes de libre disposición \\
\hline
\end{tabular}

\subsection{Superficie forestada}

La superficie total forestada desde 1994 al año 2016 ha sido de 732926 ha (tabla 5), magnitud verdaderamente relevante en el contexto de las forestaciones o reforestaciones realizadas por particulares. Como antecedente más próximo, a esta línea de ayudas, estarían las repoblaciones realizadas al amparo de la L.7/1977, de fomento a la producción forestal, donde se repoblaron 220000 hectáreas en el periodo comprendido entre 1978 y 1993 (Vadell et al., 2016).

Tabla 5. Superficie forestada por Comunidad Autónoma en cada uno de los periodos de programación.

\begin{tabular}{|c|c|c|c|c|c|}
\hline \multirow[b]{2}{*}{ Periodo de programación } & \multicolumn{5}{|c|}{ Superficie forestada (ha) } \\
\hline & 1993-99 & $2000-06$ & $2007-13$ & 2014-20 & Total \\
\hline Andalucía & 120080 & & & & 120080 \\
\hline Aragón & 4796 & 4406 & 1937 & & 11139 \\
\hline Principado de Asturias & 7737 & 2380 & & & 10117 \\
\hline Islas Baleares & 960 & 28 & & & 988 \\
\hline Islas Canarias & 1026 & 108 & & & 1134 \\
\hline Cantabria & 538 & 1083 & 95 & & 1716 \\
\hline Castilla-La Mancha & 75021 & 37226 & 3541 & 80 & 115868 \\
\hline Castilla y León & 122088 & 70231 & 42603 & 4803 & 239725 \\
\hline Cataluña & 1766 & & & & 1766 \\
\hline Comunidad Foral de Navarra & 1255 & 1502 & 252 & & 3009 \\
\hline Comunidad de Madrid & 6019 & 3061 & & & 9080 \\
\hline Comunidad Valenciana & 6635 & & & & 6635 \\
\hline Extremadura & 53333 & 13088 & 6739 & & 73160 \\
\hline Galicia & 33749 & 36830 & & & 70579 \\
\hline País Vasco & 29000 & 23605 & 98 & & 52703 \\
\hline Región de Murcia & 7893 & & & & 7893 \\
\hline La Rioja & 1487 & 5435 & 100 & 312 & 7334 \\
\hline Total & 473383 & 198983 & 55365 & 5195 & 732926 \\
\hline
\end{tabular}

Fuente: Estadística Forestal. Ministerio de Agricultura 
La evolución en el tiempo de la superficie forestada (tabla 5) muestra una tendencia claramente descendente a partir de 1999, siendo prácticamente inexistente en la actualidad. Esta tendencia está claramente marcada por el modelo de financiación adoptado, dado que las primas de mantenimiento y compensación han sido cada vez mayores y han comprometido el presupuesto para las nuevas forestaciones. Esto es particularmente evidente a partir del año 2000, como consecuencia del gran éxito que tuvo este programa en el periodo 1993-99.

Haciendo referencia a los cuatros periodos de programación que ha abarcado la FTA, el periodo de mayor actividad fue el de 1993-99, en donde se forestó el 65\% del total. Durante este periodo se alcanzaron valores anuales próximos a los registrados por el Patrimonio Forestal del Estado en la década de los años cincuenta y sesenta del siglo pasado (Vadell et al., 2016). No obstante, esta cifra quedó muy lejos de las previsiones realizadas de forestar 806593 ha (Gómez-Jover y Jiménez Peris, 1997). En los siguientes periodos, la superficie forestada representó el $27 \%, 7 \%$ y $1 \%$, respectivamente.

Analizando la superficie forestada por cada Comunidad Autónoma, destaca Castilla y León donde se ha forestado el 33\% de la superficie total, seguida por Andalucía y Castilla La Mancha donde se ha forestado el 16\% en cada una de ellas. Les siguen Extremadura y Galicia con un 10\% y País Vasco con un 7\% de la superficie total forestada. Las CC.AA. donde menos éxito ha tenido el programa han sido Cataluña, Cantabria, la Región de Murcia y la Comunidad Valenciana, donde solo se forestó durante el periodo 1993-99.

Paralelamente a la medida de forestación de tierras agrícolas, el Reglamento (CE) 1698/2005 estableció otra medida que fue la forestación de tierras no agrícolas. En el periodo 2007-13 esta medida supuso la forestación de 35251 ha (MAPA, 2013). Solo activaron esta medida cinco CC.AA., destacando la superficie forestada en Galicia con 25479 ha y Asturias con 5407 ha. En Andalucía, Aragón y La Rioja se forestaron menos de 2000 ha. Las superficies forestadas, en muchos casos, fueron tierras abandonadas.

La superficie forestada en cada Comunidad Autónoma, en el periodo 1993-99, ha mostrado una correlación directa y significativa con las siguientes variables referidas al año 1994, año de inicio de la FTA (tabla 6):

- Superficie total de cultivos.

- Superficie de cultivos herbáceos de secano.

- Superficie de barbecho de secano.

- Superficie de erial a pastos.

- Superficie de prados y pastizales.

- Superficie forestal.

No muestra correlación alguna con los valores relativos de la superficie de cultivos (SH/ST), superficie de cultivos herbáceos de secano y de barbecho $(\mathrm{SH}+\mathrm{SB} / \mathrm{ST})$ o la superficie de cultivos y prados y pastizales $(\mathrm{SC}+\mathrm{SP} / \mathrm{ST})$. Para las seis variables explicativas que han mostrado una correlación con la superficie forestada, los gráficos de dispersión permiten visualizar comportamientos muy dife- 
Tabla 6. Coeficiente de correlación de Pearson y grado de significación entre la superficie forestada en el periodo 1994-199 en cada Comunidad Autónoma y las variables explicativas del ámbito agrario referidas al comienzo del periodo (1994).

\begin{tabular}{|c|c|c|c|c|c|c|c|c|c|c|c|c|c|}
\hline & SC & SH & SB & SP & SEP & I94 & PA94 & SC/ST & SH+SB/ST & SC+SP/ST & I/SC & PA/PT & PA/SC \\
\hline FTA & $\begin{array}{c}0,885 \\
* * *\end{array}$ & $\begin{array}{c}0,883 \\
* * *\end{array}$ & $\begin{array}{c}0,734 \\
* *\end{array}$ & $\begin{array}{c}0,862 \\
* * *\end{array}$ & $\begin{array}{c}0,787 \\
* *\end{array}$ & $\begin{array}{c}0,672 \\
\text { n.s. }\end{array}$ & $\begin{array}{c}0,741 \\
\text { n.s. }\end{array}$ & $\begin{array}{c}0,239 \\
\text { n.s. }\end{array}$ & $\begin{array}{c}0,387 \\
\text { n.s. }\end{array}$ & $\begin{array}{c}0,229 \\
\text { n.s. }\end{array}$ & $\begin{array}{c}0,334 \\
\text { n.s. }\end{array}$ & $\begin{array}{c}0,405 \\
\text { n.s. }\end{array}$ & $\begin{array}{c}-0,250 \\
\text { n.s. }\end{array}$ \\
\hline
\end{tabular}

${ }^{* * *} \mathrm{p}<0,0001,{ }^{* *} \mathrm{p}<0,001$, n.s: no significativo; FTA: superficie forestada periodo 1993-99, SC: superficie total de cultivos1994; SH: superficie cultivos herbáceos de secano1994; SB: superficie de barbecho secano1994; SP: superficie de pastos y pastizales1994; SEP: superficie de erial a pastos1994 I: Producción agrícola1989; PA: población activa agraria1994; PT: población total1994; ST: SC+SP+SF

Fuente: MAPA, 2019

rentes según las CC.AA. (Fig. 1 en página siguiente). Tomando como referencia la línea de tendencia en los diferentes gráficos, se puede observar como Castilla y León y Andalucía han hecho un gran esfuerzo de forestación, mientras que Casti1la-La Mancha ha forestado menos en comparación con la superficie de terrenos aptos para la misma. Extremadura, Galicia y País Vasco han forestado superficies relativamente elevadas en comparación con la extensión de los terrenos susceptibles de ser forestados. Sin embargo, en Aragón, Cataluña, Comunidad Valenciana o la Región de Murcia, con grandes superficies susceptibles de ser forestadas, la superficie forestada ha sido mínima.

\subsection{Las especies utilizadas}

Las series disponibles de la superficie forestada por especies (MAPA 2004) no coinciden con las series de superficie total forestada por CC.AA., ya que alguna de las fuentes de información utilizadas para la corrección de esta no dispone de su desglose por especie. La información sobre las especies utilizadas se dispone con cierta precisión para el periodo 1993-99, pero no para el resto (tabla 7). No obstante, dado que en este periodo se forestó el $65 \%$ de toda la superficie, los resultados pueden ser extrapolables al resto de los periodos.

La primera consecuencia a destacar es el uso mayoritario de las frondosas entre las especies de crecimiento lento, tanto en la formación de masas puras y mixtas. Este aspecto contrasta con el uso mayoritario de las coníferas en el periodo 194084 (Vadell et al., 2016). Sin embargo, en el uso de las especies de crecimiento rápido fue mayoritario el uso de resinosas. Hay que destacar que la creación de masas puras representó el 59\%, siento el resto de masas mixtas. Por su magnitud, representó una novedad el uso de especies arbustivas o de arbóreas de carácter no dominante, al forestarse 4543 ha.

Entre las frondosas destacó el uso de Quercus ilex, con el que se forestó cerca de 175 mil hectáreas, formando masas puras y mixtas (tabla 8). Entre las coníferas destacó el uso de Pinus halepensis con más de 35 mil hectáreas de masas puras. Entre las especies de crecimiento rápido destacó el uso de Pinus radiata. 


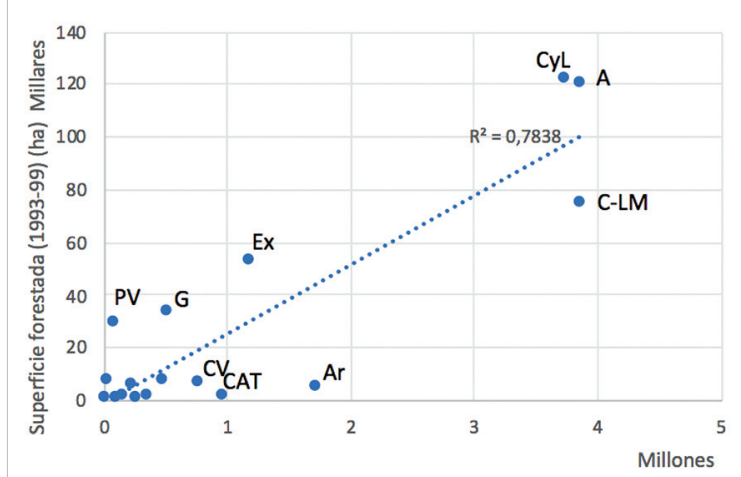

Superficie total cultivos (1994) (ha)

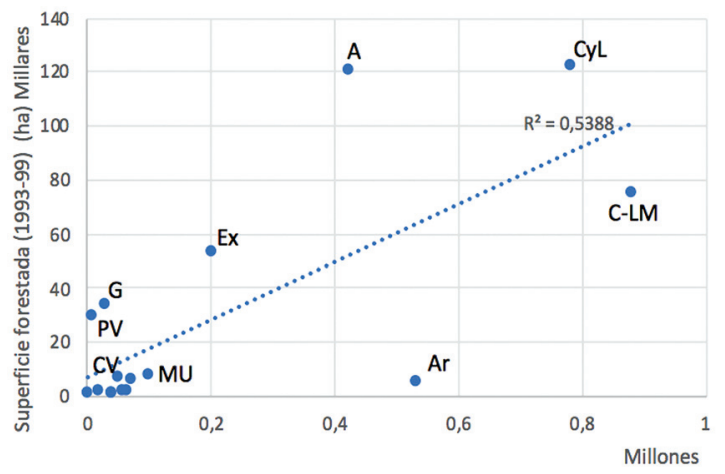

Superficie barbecho secano (1994) (ha)

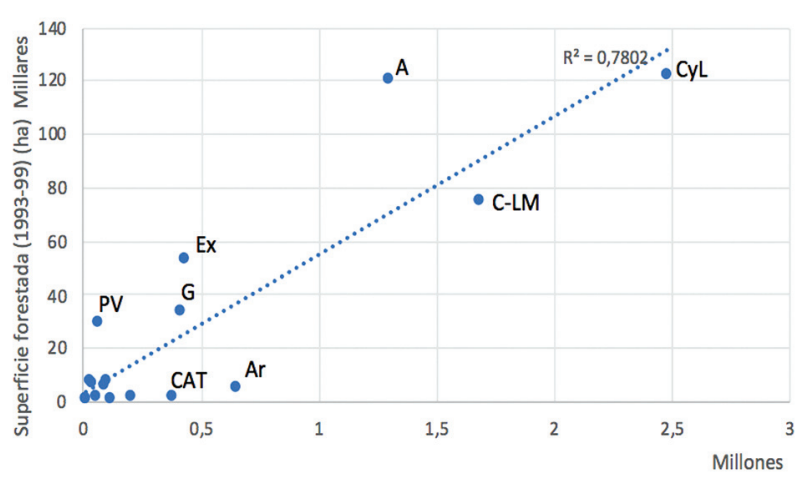

Superficie cultivo herbáceo secano (1994) (ha)

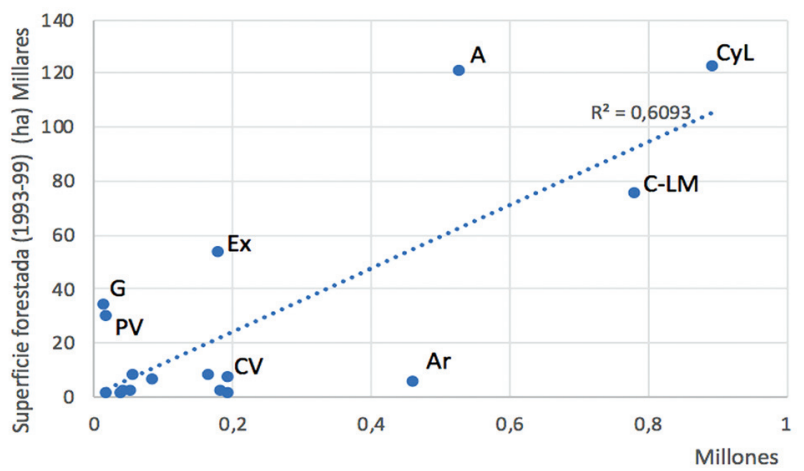

Superficie de erial a pastos (1994) (ha)

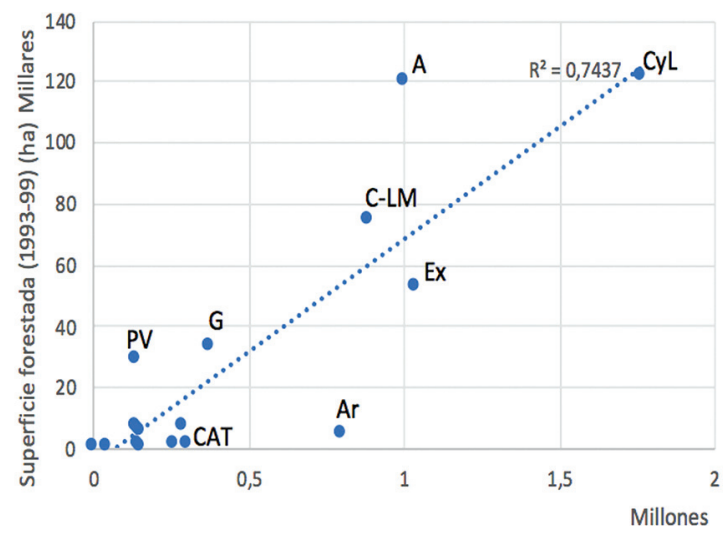

Superficie prados y pastizales (1994)(ha)

Figura 1. Gráficos de dispersión con línea de tendencia de la superficie total forestada en cada Comunidad Autónoma en el periodo 1993-99 con la superficie total de cultivos en el año 1994 (superior izquierda), la superficie de cultivos herbáceos de secano en el año 1994 (superior derecha), la superficie de barbechos de secano en el año 1994 (centro izquierda), la superficie de erial a pastos en el año 1994 (centro derecha) y la superficie de prados y pastizales en el año 1994 (inferior izquierda). Cada punto corresponde a una Comunidad Autónoma. Abreviaturas: A: Andalucía; Ar: Aragón, C-LM: Castilla-La Mancha, CyL: Castilla y León, CAT: Cataluña, CV: Comunidad Valenciana, Ex: Extremadura, MU: Región de Murcia, PV: País Vasco. 
Tabla 7. Tipo de especie utilizada en la forestación de tierras agrarias según tipo de crecimiento y composición de la masa en el periodo 1993-2013.

\begin{tabular}{|c|c|c|c|c|c|c|}
\hline \multirow[t]{2}{*}{ Crecimiento } & \multirow{2}{*}{$\begin{array}{c}\text { Composición } \\
\text { masa }\end{array}$} & \multirow[t]{2}{*}{ Tipo de especie } & \multicolumn{4}{|c|}{ Superficie forestada (ha) } \\
\hline & & & 1993-1999 & $2000-2006^{1}$ & 2007-2013 & Total \\
\hline \multirow[t]{5}{*}{ Lento } & \multirow[t]{3}{*}{ Pura } & Frondosas & 123743 & 8179 & 10233 & 142155 \\
\hline & & Coníferas & 86459 & 30916 & 27919 & 145294 \\
\hline & & $\begin{array}{l}\text { Otras arbóreas } \\
\text { y arbustivas }\end{array}$ & 4543 & - & 446 & 4989 \\
\hline & \multirow[t]{3}{*}{ Mezcla } & Frondosas & 99394 & - & 11458 & 110852 \\
\hline & & Coníferas & 18484 & - & 1049 & 19533 \\
\hline \multirow{5}{*}{ Rápido } & & Frondosas -Coníferas & 57075 & - & 20863 & 77938 \\
\hline & \multirow[t]{2}{*}{ Pura } & Frondosas & 8177 & 3983 & 2501 & 14661 \\
\hline & & Coníferas & 46049 & 13640 & 6105 & 65794 \\
\hline & Mezcla & Frondosas - Coníferas & 7264 & - & - & 7264 \\
\hline & Total & & 451188 & 56718 & 80574 & 588480 \\
\hline
\end{tabular}

' No hay datos en el periodo 2003-05.

Tabla 8. Especies más importantes utilizadas en la forestación de tierras agrarias durante el periodo 1993-2013 en la formación de masas monoespecíficas.

\begin{tabular}{lc}
\hline \multicolumn{1}{c}{ Especie } & Superficie forestada (ha) (1993-2013) \\
\hline Quercus ilex & 59633 \\
Quercus suber & 36203 \\
Olea europaea var sylvestris & 16153 \\
Ceratonia siliqua & 9604 \\
Juglans regia & 6077 \\
Castanea sativa & 4705 \\
\hline Otras frondosas & 15209 \\
Pinus halepensis & 35430 \\
Pinus nigra & 24420 \\
Pinus pinea & 24643 \\
Pinus sylvestris & 18668 \\
Pinus pinaster & 25657 \\
Otras coníferas & 16474 \\
Tetraclinis articulata & 680 \\
Tamarix gallica & 735 \\
Sorbus spp & 578 \\
\hline Otras especies arbustivas & 2995 \\
Eucalyptus spp & 8631 \\
Populus spp & 5340 \\
Quercus rubra & 689 \\
Pinus radiata & 53611 \\
\hline
\end{tabular}

${ }^{1}$ No se dispone de datos 2003-05. (MAPA 2004; MAPA 2016). 
El diseño de estas forestaciones, en cuanto al tipo de masas creadas y las especies que las componen supone un gran contraste en relación a las masas forestales creadas en el periodo 1940-84. Su composición responde a las recomendaciones dictadas por la UE para la elección de especies (UE, 2014), siendo similar a la composición de las masas creadas antes de 1940 (Vadell et al., 2016).

\subsection{Tipo de superficies forestadas}

La superficie dedicada a uso agrícola muestra un descenso continuado de la superficie dedicada a cultivos en el periodo comprendido entre 1990 y 2013, que alcanza 3 039620 millones de hectáreas (Fig. 2). Este importante descenso deja patente la existencia de un proceso de abandono agrícola que dificulta visualizar la contribución al mismo de la FTA. En este periodo, destaca, de forma especial, la pérdida de $1.2 \mathrm{mi}-$ llones de hectáreas de cultivos entre 1993 y 1994, año de comienzo de la FTA. Esta reducción es menor en el caso de la superficie dedicada a prados naturales y pastizales que, durante este periodo, redujeron su superficie en 367827 ha. Paralelamente, la superficie forestal aumentó en 3597290 ha (MAPA, 2019).

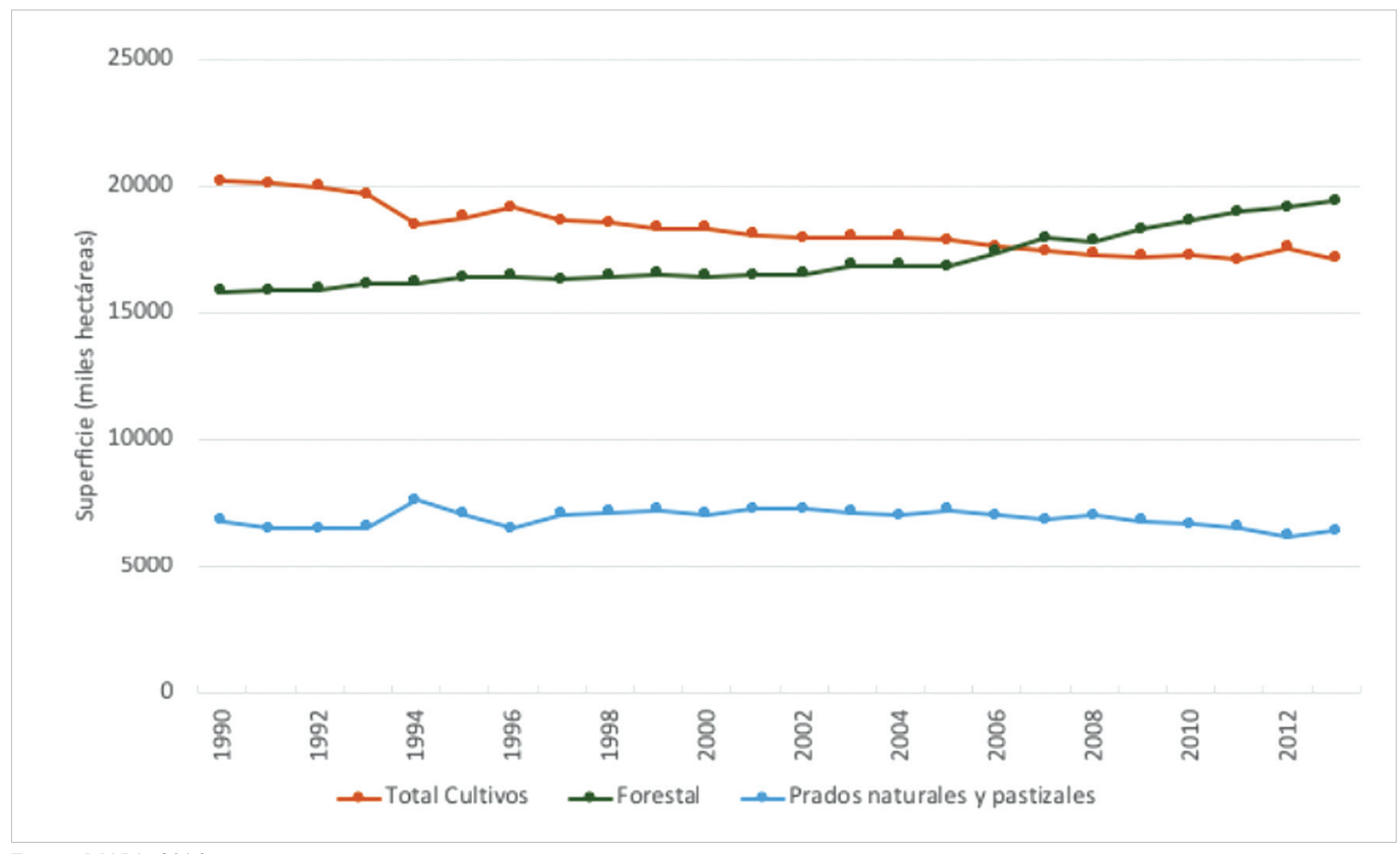

Fuente: MAPA, 2016

Figura 2. Evolución de la superficie en España dedicada a cultivos, prados naturales, pastizales y terrenos forestales en el periodo 1990-2013.

La magnitud de la superficie que ha dejado de cultivarse en este periodo, cuatro veces superior a la forestada, indica que el abandono agrícola es el proceso que condiciona en la actualidad los cambios de uso de la tierra. Por tanto, cabe pensar 
que en cuanto a la retirada de tierras de cultivo la forestación no era una medida necesaria, pero podría ser si de interés al implicar que el nuevo uso forestal de estas tierras tenía un carácter irreversible.

No existe una estadística en todo el periodo estudiado que indique el tipo de tierras objeto de forestación. Solo se conoce con precisión la tipología de superficies en el periodo 1993-99, siendo los eriales a pastos los de mayor representación (34\%) (tabla 9). Que este tipo de tierras fueran las mayormente forestadas durante el primer periodo contrasta con los tipos de tierras agrarias objeto de forestación recogidos en el Reglamento 2080/92, donde los eriales a pastos eran la última clase, no justificándose, en este caso, la existencia de una prima compensatoria de pérdida de rentas, por la falta de rentas en los mismos.

Tabla 9. Superficie forestada según el tipo de tierra agraria en el periodo 1993-1999 .

\begin{tabular}{lcc}
\hline \multicolumn{1}{c}{$\begin{array}{c}\text { Tipo de } \\
\text { tierra agraria }\end{array}$} & $\begin{array}{c}\text { Superficie } \\
\text { forestada (ha) }\end{array}$ & $\begin{array}{c}\text { Porcentaje respecto a la superficie } \\
\text { total forestada (\%) }\end{array}$ \\
\hline Tierras ocupadas por cultivos herbáceos & 100419 & 22 \\
Barbechos y otras tierras no ocupadas & 42676 & 9 \\
Huertos familiares & 722 & 0 \\
Tierras ocupadas por cultivos leñosos & 19127 & 4 \\
Prados naturales & 3293 & 1 \\
Pastizales & 66495 & 15 \\
Montes de alcornocal & 1940 & 0 \\
Monte abierto y dehesas & 61984 & 14 \\
Eriales a pastos & 154508 & 34 \\
\hline \multicolumn{1}{c}{ Total } & 451164 & \\
\hline
\end{tabular}

${ }^{1}$ No se dispone de datos 2003-05. (MAPA 2004; MAPA 2016).

Este hecho fue puesto en evidencia por un informe del Tribunal Cuentas (UE, 2005) en el que se indicaba, además, la dudosa verificación que hicieron los Estados miembros, entre ellos España, de la información que aportaba el beneficiario. Esta información no se comprobaba con las declaraciones que este hubiera hecho los años anteriores en el sistema SIGPAC. En este sentido, existe constancia de que algunas de las tierras que se acogieron a esta medida estaban abandonadas desde hacía muchos años. A este aspecto contribuyó el hecho de que en España se estableció como plazo para certificar un uso agrario de la tierra el de 10 años, periodo cinco veces superior al de otros Estados miembros.

El impacto que ha tenido la forestación de tierras agrarias en las diferentes Comunidades Autónomas es difícil de apreciar a partir de la variación de la superficie total producida en los diferentes cultivos o usos de la tierra. Así, para el periodo 1994-99 (tabla 10), el comportamiento que ha tenido esta variación ha sido muy dispar, aumentándose en algunos casos la superficie dedicada a cultivos, a eriales a pastos o disminuyendo incluso la superficie forestal. Debe destacarse como en el Principado de Asturias, donde la superficie forestada en el periodo 1993-99 repre- 
sentó el 26\% de la superficie total de cultivos en 1994, aumentó la superficie total de cultivos y la de pastos en el periodo 1994-99. Es evidente, por tanto, el poco impacto de la forestación de tierras agrarias en la variación de la superficie de los diferentes usos de la tierra.

Tabla 10. Variación de la superficie (hectáreas) por tipo de cultivos entre 1994 y 1999.

\begin{tabular}{lcccccc}
\hline & $\begin{array}{c}\mathbf{S C}_{1994} \\
\mathbf{- S C}_{1999}\end{array}$ & $\begin{array}{c}\mathbf{S H}_{1994} \\
\mathbf{- S H}_{1999}\end{array}$ & $\begin{array}{c}\mathbf{S P}_{1994} \\
\mathbf{- S P}_{1999}\end{array}$ & $\begin{array}{c}\mathbf{S F}_{1999} \\
\mathbf{- S F}_{1994}\end{array}$ & $\begin{array}{c}\mathbf{S E P}_{1994} \\
\mathbf{- S E P}_{1999}\end{array}$ & $\begin{array}{c}\mathbf{F T A}_{(1993-999} \\
\text { / SC }_{1994}(\%)\end{array}$ \\
\hline Andalucía & -20600 & 60056 & -58105 & -46266 & 10051 & 3.1 \\
Aragón & -56942 & -76938 & 90213 & -82741 & -128373 & 0.3 \\
Principado de Asturias & -866 & -304 & -18289 & 24838 & 17614 & 26.2 \\
Illes Balears & 76320 & 55030 & -3665 & 30076 & 19648 & 0.4 \\
Islas Canarias & 58772 & 4610 & 8980 & 70751 & -10922 & 1.0 \\
Cantabria & 3527 & 3240 & -6922 & 7067 & 2409 & 3.3 \\
Castilla-La Mancha & -148227 & -88355 & 160617 & 40958 & 16231 & 1.9 \\
Castilla y León & 37824 & 12658 & 129004 & 6904 & -146065 & 3.3 \\
Cataluña & 58659 & 33630 & 32912 & 47109 & -42830 & 0.2 \\
Comunidad Foral de Navarra & 14416 & 5976 & -8038 & 598 & -3050 & 0.3 \\
Comunidad de Madrid & 8385 & 5079 & 11216 & 5630 & -857 & 2.5 \\
Comunitat Valenciana & 44193 & -16189 & 30161 & 170523 & 103719 & 0.8 \\
Extremadura & -139586 & -4024 & 95537 & 102551 & 97005 & 4.5 \\
Galicia & 142723 & 118413 & -83264 & 8857 & 1111 & 6.4 \\
País Vasco & -865 & 847 & -18735 & -26505 & -1174 & 31.8 \\
Región de Murcia & 28464 & 24339 & -30240 & -3739 & 248 & 1.6 \\
La Rioja & 9416 & 10309 & 27942 & 19476 & -16866 & 0.9 \\
\hline \multicolumn{1}{c}{ Total } & $\mathbf{1 1 5 6 1 3}$ & $\mathbf{1 4 8 3 7 7}$ & $\mathbf{3 5 9 3 2 4}$ & $\mathbf{3 7 6 0 8 7}$ & $\mathbf{- 8 2 ~ 1 0 1}$ & \\
\hline
\end{tabular}

SC: Total de superficie de cultivos, SH: superficie de cultivos herbáceos de secano; SP: superficie de prados y pastizales; SF: superficie forestal; SEP: superficie de erial a pastos; FTA: superficie forestada periodo 1993-99.

Llama la atención como la superficie de eriales a pastos aumentó en el periodo 1994-99, probablemente como consecuencia del abandono de cultivos herbáceos o de pastos.

\subsection{Superficie media de las zonas forestadas}

El tamaño de las superficies objeto de forestación, en cuanto a su superficie mínima y máxima, podría dar una idea de la entidad real de las masas creadas y su papel en la fragmentación y conectividad de los ecosistemas agrícolas y forestales. No existe una estadística que haga referencia a esta variable, pero Picard (2001) ofrece para el periodo 1993-99 una superficie media de 11.96 ha. Esta cifra presenta una gran variabilidad, oscilando entre 2 ha en la Cornisa Cantábrica y 52 ha en Andalucía y Extremadura. Para el periodo 2000-06, se estimó a partir de la super- 
ficie forestada y del número de beneficiarios, obteniendo un valor de 11.6 ha, muy similar al del periodo anterior (TRAGSATEC, 2008).

Para el periodo 2007-13, los datos de los que se dispone son de los informes finales de las diferentes CC.AA. y, siguiendo el mismo procedimiento que para el periodo anterior, se han obtenido valores medios de 2 ha en La Rioja, 3.7 en Aragón, 4 en el País Vasco, 6.7 en Castilla y León u 8.8 ha en Castilla La Mancha.

\section{Conclusiones}

1. Este régimen de ayudas se ha contemplado durante cuatro periodos de programación, primero en el contexto de la Política Agrícola Comunitaria y luego dentro de las políticas de desarrollo rural. Los objetivos con ella perseguidos han variado en los diferentes marcos normativos.

2. La elevada superficie forestada hace de la FTA la medida o ayuda de mayor impacto en España entre los propietarios particulares. No obstante, ante el grado de discrepancia que existe entre los valores oficiales publicados y las series facilitadas para la realización de este estudio, por parte de algunas Comunidades Autónomas, este valor podría no representar la realidad de la superficie forestada. La Comunidad Autónoma que más ha forestado ha sido Castilla y León, donde la FTA todavía permanece activa.

3 . El $65 \%$ de la superficie total forestada se produjo en el primer periodo de programación, en donde esta ayuda fue activada por todas las Comunidades Autónomas. No ocurrió así en el resto de los periodos.

4. La mitad de la superficie forestada se realizó con masas monoespecíficas, teniendo similar superficie la realizada con frondosas y coníferas. La superficie forestada con especies de crecimiento rápido representó un $15 \%$ de la superficie total. El resto de la superficie se forestó creando masas mixtas, donde fue predominante el uso de las frondosas. La especie más utilizada fue Quercus ilex.

5. Las superficies donde más se forestó son las correspondientes a cultivos herbáceos de secano y las de eriales a pastos, aunque no se observa un impacto de la forestación en los cambios de usos de la tierra.

6. La superficie media forestada por beneficiario es de $12 \mathrm{ha}$, para los dos primeros periodos de programación, aunque con gran variabilidad según la Comunidad Autónoma.

\section{Bibliografía}

Álvarez, P.; 2004. Viveros forestales y uso de planta en repoblación en Galicia. Tesis doctoral. Universidad de Santiago de Compostela.

CACAN, 2019. Forestación de tierras agrarias. Comunicación personal. Dirección General de Protección de la Naturaleza del Gobierno de Canarias. 
Gómez-Jover, F.; Jiménez Peris, F.J.; 1997. Forestación de tierras agrícolas. Madrid: Ministerio de Agricultura, Pesca y Alimentación.

González-Botía, M.A., 2017. Forestaciones en tierras agrarias de la Región de Murcia. Cambios medioambientales y paisajísticos. Tesis doctoral. Universidad de Murcia.

JCLM, 2012. Estadística forestación tierras agrarias. http://www.castillalamancha.es/sites/ default/files/documentos/pdf/20120917/estadisticas_forestacion.pdf. Accessed. 1 December 2018.

JCyL, 2018. Estadística de la forestación de tierras agrarias. Valladolid.

MAPA, 2004. Forestación tierras agrarias. Comunicación personal.

MAPA, 2006. Forestación de tierras agrícolas. Madrid: MAPA Dirección General de Desarrollo Rural.

MAPA, 2013. Programas de Desarrollo Rural. Periodo de programación 2007-13. Accessed. 15 June 2018.

MAPA, 2016. Anuarios estadística forestal. https:/www.mapa.gob.es/es/desarrollo-rural/ estadisticas/forestal_anuarios_todos.aspx. Accessed. 1 July 2018.

MAPA, 2019. Anuarios estadística agraria. https:/www.mapa.gob.es/es/estadistica/temas/ default.aspx. Accessed. 5 April 2019.

Picard, O.; 2001. Evaluation of the Community aid scheme for forestry measures in agriculture of Regulation No 2080/92. Auzeville.

Rodríguez Martínez, A.; 1993. La forestación de tierras agrícolas en Castilla-La Mancha. Foresta, 47-48, 140-145.

Sequeda, M.M.; 2017. Forestación de tierras agrarias en Extremadura. In: SECF (ed.), 7 Congreso Forestal Español. Plasencia: Sociedad Española de Ciencias Forestales. http: //secforestales.org/publicaciones/index.php/congresos_forestales/article/view/19381

TRAGSATEC, 2008. Evaluación final del Programa de Desarrollo Rural para las medidas de acompañamiento en España 2000-2006. Madrid.

UE, 2005. Informe especial 9/2004 del Tribunal de Cuentas sobre las medidas forestales en el marco de la política de desarrollo rural, acompañado de las respuestas de la Comisión. Unión Europea.

UE, 2014. Directrices de la Unión Europea aplicables a las ayudas estatales en los sectores agrícola y forestal y en zonas rurales de 2014 a 2020. Unión Europea.

Vadell, E.; de-Miguel, S.; Pemán, J.; 2016. Large-scale reforestation and afforestation policy in Spain: A historical review of its underlying ecological, socioeconomic and political dynamics. Land Use Policy 55, 37-48. http://doi.org/10.1016/j.landusepol.2016. 03.017 